\title{
A severe complication of crack cocaine use
}

\author{
Gokul Vidyasankar MD¹, Carolina Souza MD², Chi Lai MD³, Sunita Mulpuru MD
}

G Vidyasankar, C Souza, C Lai, S Mulpuru. A severe complication of crack cocaine use. Can Respir J 2015;22(2):77-79.

The present report describes a 48-year-old woman with a history of recurrent 'crack' cocaine use, who developed progressive shortness of breath over a period of years. Serial imaging revealed progressive interstitial fibrosis secondary to recurrent alveolar hemorrhage and inflammation from crack cocaine. The present case serves as a reminder of the numerous sequelae of crack cocaine use, highlighting one particularly severe outcome.

Key Words: Alveolar hemorrhage; Crack lung; Cocaine; Interstitial lung disease

\section{Learning objectives}

- To recognize the array of pulmonary complications associated with crack cocaine use.

CanMEDS competency: Medical Expert

Pretest

- What is crack cocaine?

- What are the pulmonary complications of smoking crack cocaine?

\section{CASE PRESENTATION}

A 48-year-old woman initially presented with subacute dyspnea and hemoptysis in 2004. She experienced some associated weight loss and intermittent fevers over a period of six months. She denied any infectious contacts or tuberculosis risk factors. Medical history included asthma and previous venous thrombosis treated with warfarin. On social inquiry, the patient was a civil servant without occupational exposures, and had a 20 pack-year history of smoking.

On presentation, the patient was hemodynamically stable, with an oxygen saturation of $94 \%$ on room air. Cardiac and respiratory examinations were normal. Complete blood count revealed a mild leukocytosis and anemia. Initial international normalized ratio was elevated (3.6), but remained between 1.0 and 2.0 for the remainder of the admission. Renal function and urinalysis were normal. In addition, a connective tissue panel and HIV serology were negative. Chest $\mathrm{x}$-ray revealed symmetrical bilateral patchy opacities. A computed tomography (CT) scan of the thorax revealed bilateral symmetrical central ground-glass opacities with some centrilobular nodules, consistent with alveolar hemorrhage or atypical infection (Figure 1).

Fibreoptic bronchoscopy was then performed, which did not reveal any evidence of active hemorrhage. Bronchoalveolar lavage was negative for culture, including mycobacterial culture. Subsequently, the patient underwent an open-lung biopsy for diagnosis, which revealed mild peribronchiolar inflammation with areas of organizing pneumonia and frequent hemosiderin-laden macrophages. There was no evidence of capillaritis. The overall process was believed to be consistent with organizing pneumonia (Figure 2). The patient improved and was discharged, but was lost to follow-up.

\section{Une grave complication de la consommation de crack}

Le présent rapport décrit le cas d'une femme de 48 ans ayant une histoire de consommation récurrente de crack, atteinte d'un essoufflement chronique qui s'est détérioré sur plusieurs années. L'imagerie sérielle a révélé une fibrose interstitielle progressive secondaire à une hémorragie alvéolaire récurrente et à une inflammation causée par le crack. Ce cas rappelle les nombreuses séquelles de la consommation de crack et en fait ressortir une conséquence particulièrement grave.

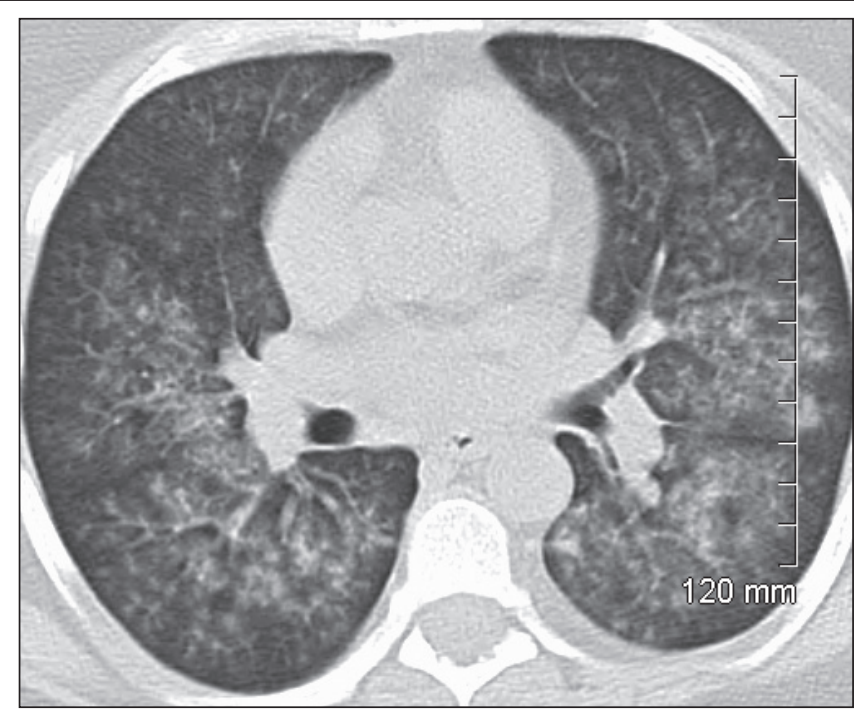

Figure 1) Chest computed tomography at initial presentation demonstrating bilateral symmetrical airspace disease in a predominant central distribution with multiple acinar opacities and centrilobular nodules in the right lower lobe

In 2007, she returned with recurrent hemoptysis and a similar clinical presentation. At that time, her oxygen saturation was reduced (88\%), with a mild decrease in hemoglobin level. Connective tissue workup was, again, negative, and bronchoscopy revealed evidence of old blood but no active hemorrhage. Once again, bronchoalveolar lavage was negative for culture. A CT scan of the thorax at that time revealed bilateral diffuse ground-glass opacities, now with associated mild fibrosis (Figure 3). Radiographically, it was believed that this was more consistent with recurrent alveolar hemorrhage and not organizing pneumonia. On further questioning, the patient eventually admitted to intermittent use of crack cocaine, which she also had been using at the time of initial presentation in 2004. Once again, she improved during admission and was discharged with a strong warning against any further cocaine use.

\footnotetext{
${ }^{1}$ Division of Respirology, Department of Medicine; ${ }^{2}$ Department of Radiology; ${ }^{3}$ Department of Anatomic Pathology, University of Ottawa, Ottawa, Ontario

Correspondence: Dr Gokul Vidyasankar, Division of Respirology, Department of Medicine, University of Ottawa, 501 Smyth Road, Box 211,

Ottawa, Ontario K1H 8L6. Telephone 613-794-2383, e-mail gokul.vidyasankar@gmail.com
} 


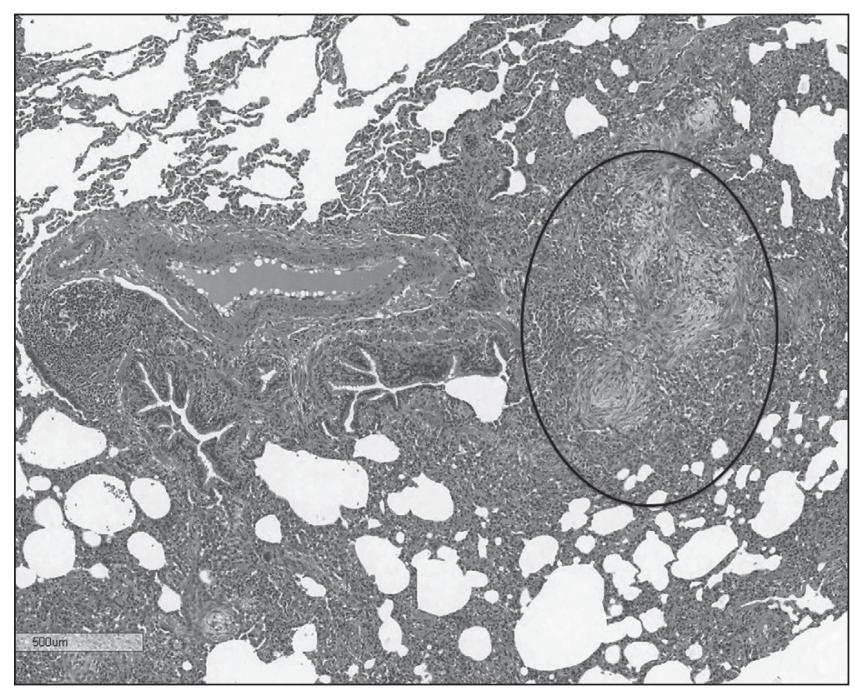

Figure 2) Mild peribronchiolar chronic inflammation and scattered foci of organizing pneumonia (circle) (hematoxylin and eosin stain, original magnification $\times 40$ )

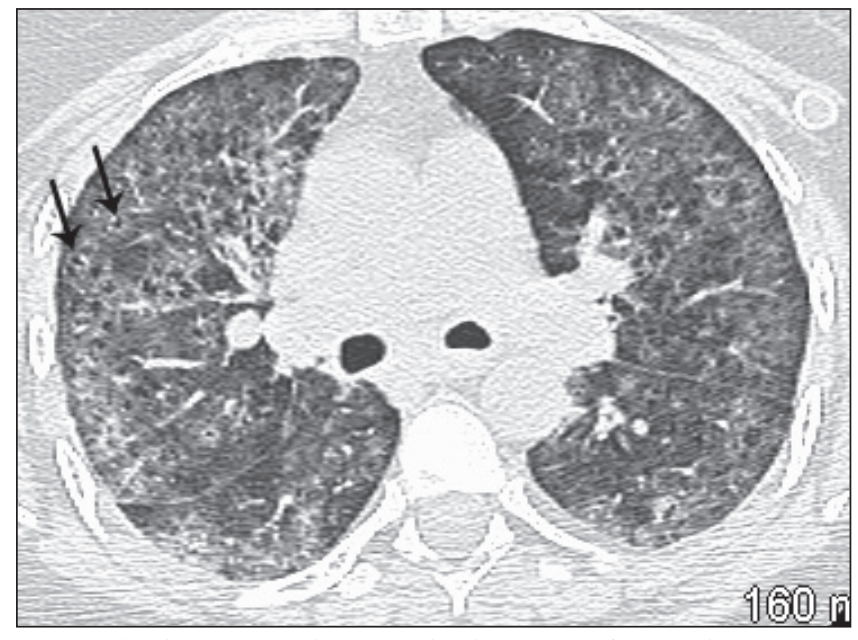

Figure 3) Chest computed tomography three years after initial presentation demonstrating bilateral diffuse ground-glass and peripheral reticular opacities. The presence of reticulation and traction bronchiolectasis (arrows) indicates early interstitial fibrosis

Unfortunately, this patient continued to smoke crack cocaine, leading to another similar admission in 2010. In March 2014, this patient was admitted to the authors' hospital with subacute progression of dyspnea on exertion. By that time, she was on long-term oxygen therapy, with significant exertional desaturation. She persistently denied any cocaine use since 2010, and had no recent history of hemoptysis. A high-resolution CT of the thorax was performed, which identified diffuse coarse reticulation with traction bronchiectasis and minimal ground-glass opacities (Figure 4). These findings were compatible with severe interstitial fibrosis.

Given the serial images over the years, it was believed that this patient's interstitial lung disease was secondary to recurrent episodes of alveolar hemorrhage as a result of crack cocaine use. The patient's oxygenation did not improve despite an empirical trial of antibiotics and diuresis; she was eventually discharged home requiring $5 \mathrm{~L}$ of oxygen via nasal accumulator. Her oxygen demands increased in subsequent months, and a contrast echocardiogram performed during a later admission confirmed severe pulmonary hypertension with a right-toleft shunt via a patent foramen ovale. Eventually, the patient was transferred to a palliative care facility for symptom management and end-of-life care.

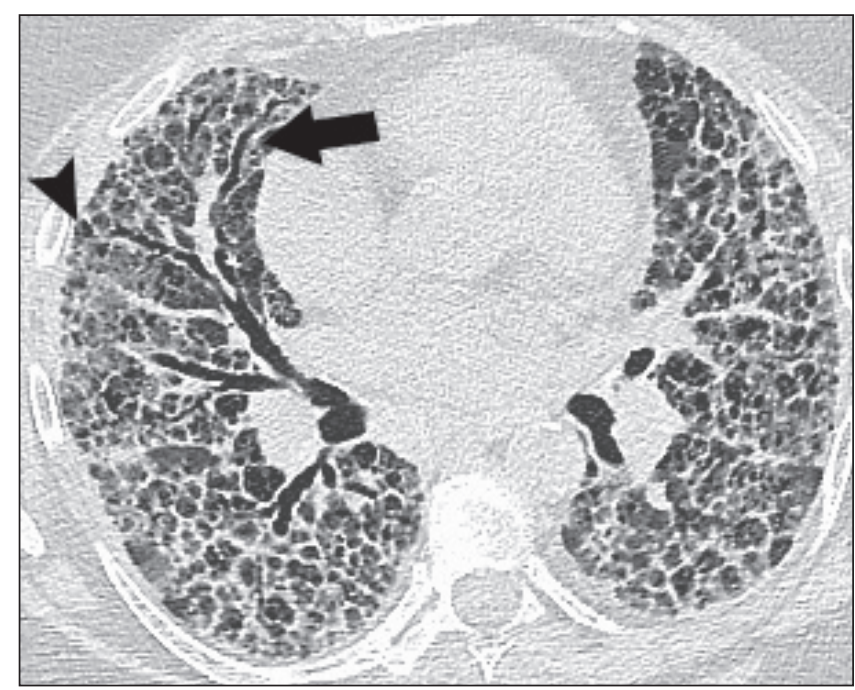

Figure 4) Chest computed tomography scan 10 years after initial presentation demonstrating diffuse coarse reticular opacities with minimal groundglass opacities, traction bronchiectasis (arrow) and early honeycombing formation (arrowhead), compatible with severe interstitial fibrosis

\section{DISCUSSION}

Cocaine is one of the most popular illicit drugs used worldwide, with numerous adverse health consequences. Due to its highly addictive potential, it continues to be a major issue globally. Extracted from the coca plant, cocaine hydrochloride can be used in several ways, including nasal insufflation, injection and via the modified form of crack cocaine. Typically, crack cocaine is made by boiling cocaine hydrochloride in a bicarbonate solution and collecting the precipitate by filtration or via a solvent (ether or alcohol). This converts the ionic form of the drug to its chemical free base form, which is heat stable and allows for it to be smoked. This process is known as 'free basing' (1). Of all the modalities to use cocaine, smoking crack provides one of the fastest rates of systemic absorption, entering the central nervous system circulation within $6 \mathrm{~s}$ to $8 \mathrm{~s}(1)$.

Several pulmonary complications have been reported with the use of crack cocaine, including noncardiogenic pulmonary edema, thermal injury, pneumothorax, alveolar hemorrhage, pulmonary infarction, eosinophilic pneumonia and organizing pneumonia $(1,2)$. These consequences may be due to the direct action of cocaine itself, or as a result of adulterants, contaminants and solvents associated with the processing of the drug. Chronic pulmonary complications include interstitial lung disease (primarily due to inhalation of adulterants), pulmonary hypertension, emphysema and airway stenosis (3).

A syndrome known as 'crack lung' has also been described, which includes a constellation of signs and symptoms including chest pain, fever, dyspnea, hemoptysis, melanoptysis, hypoxemia and respiratory failure. Chest imaging usually reveals diffuse interstitial and alveolar parenchymal opacities, occasionally with small pleural effusions $(2,4)$. In reality, this is usually due to several concurrent complications, and lung biopsy may reveal findings of diffuse alveolar damage, alveolar hemorrhage and eosinophilic infiltration $(2,4)$.

Cocaine use can also lead to several nonpulmonary complications. Cardiovascular effects include myocardial infarction, arrhythmias, tachycardia and hypertension. Neurological consequences may include headache, seizures, stroke and cerebral atrophy over the long term. Gastrointestinal effects may include perforation and ischemic colitis. Finally, acute kidney injury may occur as a result of cocaine-induced rhabdomyolysis (5).

Diagnosis can be challenging, given that the clinical features and chest imaging are nonspecific and can mimic several other processes. A thorough history and physical examination, along with a high index of suspicion, are required to arrive at the diagnosis. Bilateral burn 
marks on the thumbs may suggest use of a crack pipe. Urine toxicology can be helpful for identifying recent cocaine use, remaining positive for days to weeks depending on the extent of usage $(2,3)$.

Management is largely supportive, and most patients with crack lung improve within three to 14 days. If eosinophilic or organizing pneumonia is suspected, glucocorticoids may be beneficial (2).

In our patient, it was believed that her repeated use of crack cocaine led to recurrent episodes of pulmonary hemorrhage. The patient remained on warfarin throughout the clinical course; although this may have precipitated these episodes, hemorrhage is a documented consequence of crack cocaine use even without coagulopathy (1). This recurrent hemorrhage, in addition to effects of possible adulterants in the cocaine, eventually resulted in severe end-stage interstitial lung disease. The present case highlights one of the severe pulmonary complications of cocaine use, and reminds physicians to keep illicit drug use in the differential when evaluating a patient with undiagnosed airspace disease.

\section{REFERENCES}

1. Haim DY, Lippmann ML, Goldberg SK, Walkenstein MD. The pulmonary consequences of crack cocaine. Chest 1995;107:233-40.

2. Hui P, Walker B, Levy RD. Patient with fever, hypoxemia, and pulmonary consolidations. Chest 2012;142:1348-51.

3. Restrepo CS, Carrillo JA, Martínez S, Ojeda P, Rivera AL, Hatta A. Pulmonary complications from cocaine and cocaine-based substances: Imaging manifestations. Radiographics 2007;27:941-56.

\section{Post-test}

- What is crack cocaine?

Crack cocaine is made by boiling cocaine hydrochloride in a solution (usually sodium bicarbonate) and extracting the precipitate, a process know as free basing. This precipitate is heat stable and can then be smoked $(1,3)$.

- What are the pulmonary complications of smoking crack cocaine? Smoking crack cocaine can lead to an array of pulmonary complications, including noncardiogenic pulmonary edema, thermal injury, pneumothorax, alveolar hemorrhage, pulmonary infarction, eosinophilic pneumonia and organizing pneumonia $(1,2)$.
4. de Almeida RR, de Souza LS, Mançano AD, et al. High-resolution computed tomographic findings of cocaine-induced pulmonary disease: A state of the art review. Lung 2014;192:225-33.

5. Warner EA. Cocaine abuse. Ann Intern Med 1993;119:226-35. 


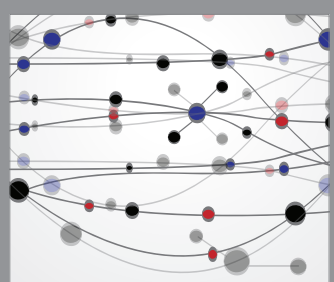

The Scientific World Journal
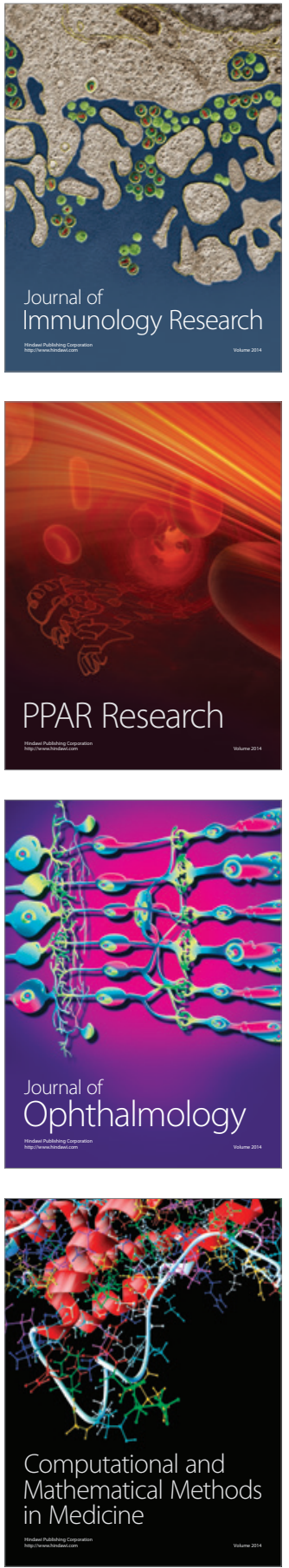

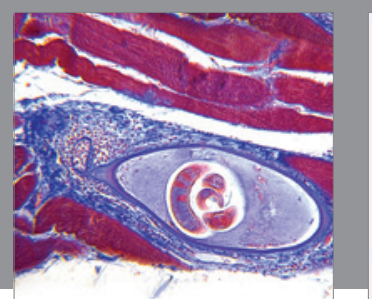

Gastroenterology Research and Practice

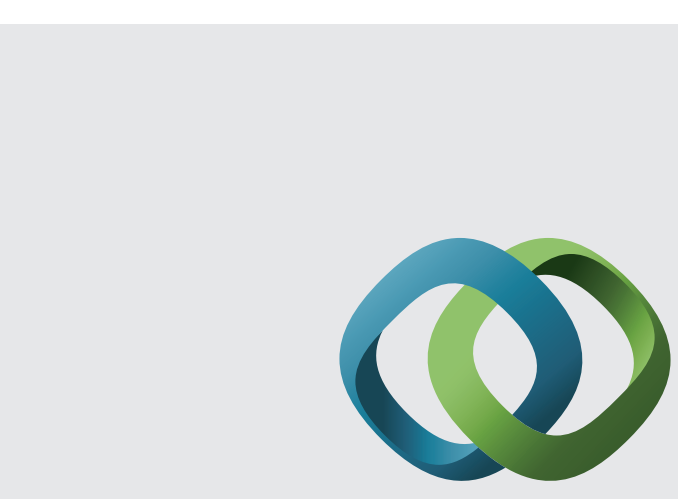

\section{Hindawi}

Submit your manuscripts at

http://www.hindawi.com
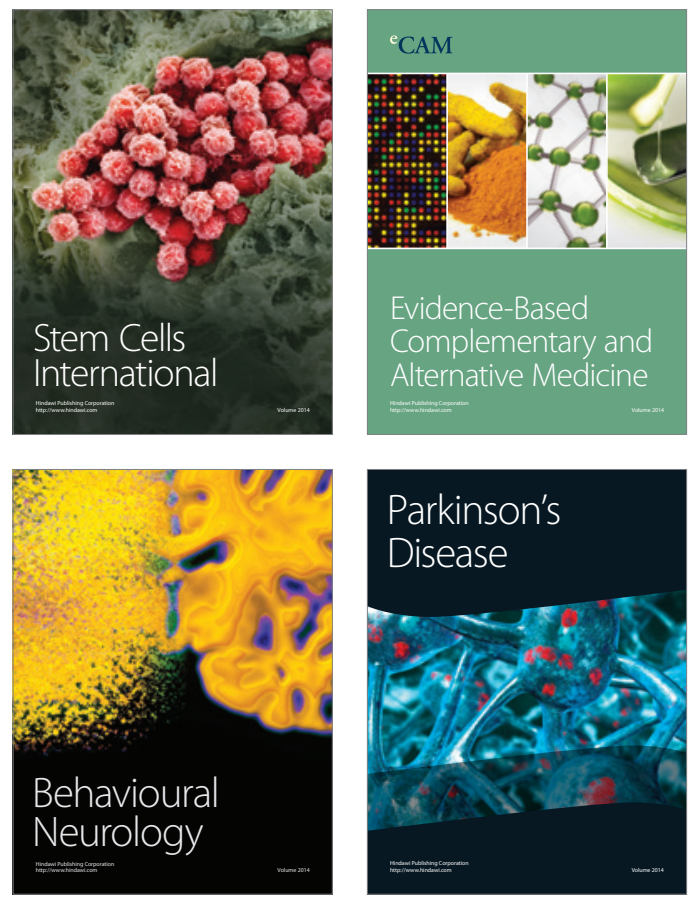
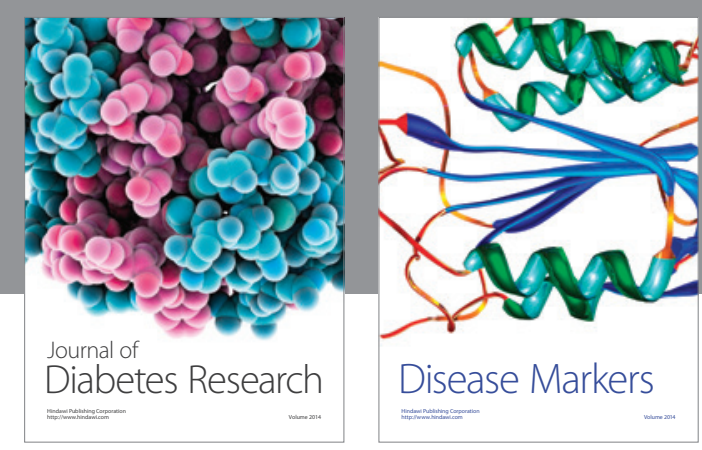

Disease Markers
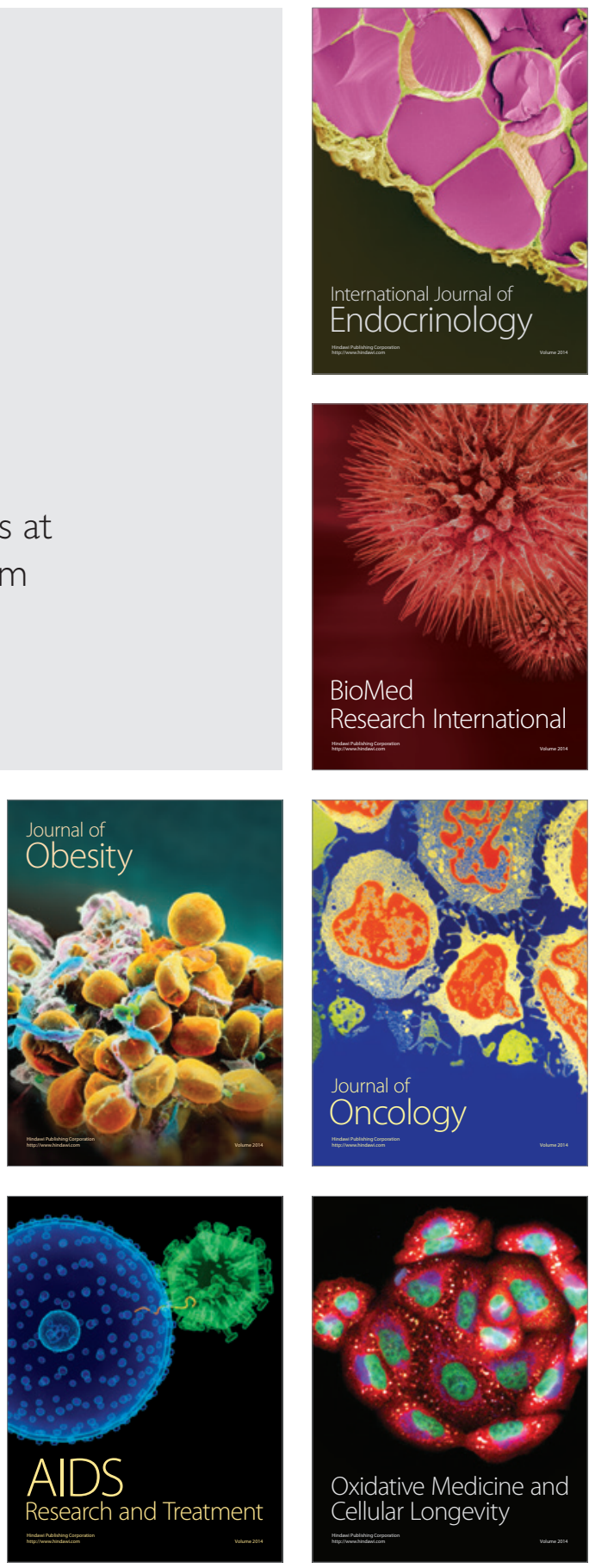